\title{
Improved Whole-Genome Sequence of Fusarium meridionale, the Fungal Pathogen Causing Fusarium Head Blight in Rice
}

\begin{abstract}
Xin Liu, ${ }^{1,2}$ Xin Fang, ${ }^{1,2}$ Fangwei $\mathrm{Yu}^{3}$ Shuang Wang, ${ }^{1,2}$ Zhichao Zhang, ${ }^{4}$ Kainan $\mathrm{Li}^{4}{ }^{4}$ Wenwu Ye, ${ }^{4}$ Yin-Won Lee, ${ }^{2,5}$ Sherif Ramzy Mohamed, ${ }^{6}$ Fei Dong, ${ }^{2, \dagger}$ Jianhong $\mathrm{Xu},{ }^{1,2, \dagger}$ and Jianrong Shi ${ }^{1,2, \dagger}$

${ }^{1}$ School of Food and Biological Engineering, Jiangsu University, Zhenjiang 212013, Jiangsu, China

2 Jiangsu Key Laboratory for Food Quality and Safety-State Key Laboratory Cultivation Base, Ministry of Science and Technology/Key Laboratory for Control Technology and Standard for Agro-product Safety and Quality, Ministry of Agriculture and Rural Affairs/Key Laboratory for Agro-product Safety Risk Evaluation (Nanjing), Ministry of Agriculture and Rural

Affairs/Collaborative Innovation Center for Modern Grain Circulation and Safety/Institute of Food Safety and Nutrition, Jiangsu Academy of Agricultural Sciences, Nanjing 210014, Jiangsu, China

${ }^{3}$ Jiangsu Key Laboratory for Horticultural Crop Genetic Improvement/Institute of Vegetable Crops, Jiangsu Academy of Agricultural Sciences, Nanjing 210014, China

${ }^{4}$ Department of Plant Pathology, Nanjing Agricultural University, Nanjing, Jiangsu 210095, China

${ }^{5}$ School of Agricultural Biotechnology, Seoul National University, Seoul 08826, Republic of Korea

${ }^{6}$ Department of Food Toxicology and Contaminant, National Research Centre of Egypt, Giza 12411, Egypt
\end{abstract}

\section{Abstract}

Members of the Fusarium graminearum species complex (FGSC) cause extensive yield losses in cereal production worldwide, and food safety concerns due to the accumulation of Fusarium toxins in infected grains. Among these pathogens, F. meridionale is responsible for Fusarium head blight of wheat and rice, ear and stalk rot of maize, and pod blight of soybean. Here, we present an improved genome assembly of $F$. meridionale strain SR5 isolated from rice in China based on PacBio long-read sequencing and Illumina short-read sequencing technology. The assembled genome of SR5 has a total size of $36.82 \mathrm{Mb}$, an $\mathrm{N}_{50}$ scaffold length of $7.82 \mathrm{Mb}$, nine scaffolds, and encodes 12,409 predicted genes. These high-quality data expand FGSC genomic resources and provide a valuable resource for better understanding their genetic diversity and the molecular basis of pathogenesis, which will facilitate the development of an effective control strategy.

\section{Genome Announcement}

Members of the Fusarium graminearum species complex (FGSC) are the major causal agents of Fusarium head blight (FHB) in wheat, barley, rice, and other small-grained crops, as well as ear and stalk rot in maize, which leads to economic losses and contamination by trichothecene mycotoxins. FGSC is composed of at least 16 species, and its species composition is host and location dependent. FGSC members are capable of producing various B-type trichothecenes, the most prevalent of which include deoxynivalenol (DON) and its acetylated derivatives, 3-acetyl deoxynivalenol (3-ADON) and 15- acetyl deoxynivalenol
†Corresponding authors: J. Shi; jianrong63@126.com, J. Xu; xujianhongnj@126.com, and F. Dong; feidong1985@126.com

The author(s) declare no conflict of interest.

Accepted for publication 16 September 2021.
Funding

This work was supported by grants from the National Key R\&D Program of China (2018YFE0206000), the National Natural Science Foundation of China (31772118), the Natural Science Foundation of Jiangsu Province (BK20181246), the Jiangsu Agriculture Science and Technology Innovation Fund (CX(21)1005), and the Key R\&D Project for Tibet Autonomous Region of China (XZ202001ZY0038N).

\section{Keywords}

Fusarium meridionale, genome assembly, long-reading sequencing, rice pathogen 
Table 1. Overview of the genome assembly features of five Fusarium meridionale strains ${ }^{\mathrm{a}}$

\begin{tabular}{|c|c|c|c|c|c|}
\hline Strain & SR5 & NR28721 & NR28723 & Fmer152 & CBS 110249 \\
\hline Genome size $(\mathrm{Mb})$ & 36.82 & 36.50 & 36.40 & 37.05 & 36.70 \\
\hline Scaffolds number & 9 & 676 & 287 & 694 & 30 \\
\hline Scaffold $\mathrm{N}_{50}$ length (Mb) & 7.81 & 0.11 & 0.39 & 0.22 & 2.44 \\
\hline Illumina coverage & $190 x$ & $51 \times$ & $32 x$ & $32 x$ & $67 x$ \\
\hline PacBio coverage & $388 x$ & ND & ND & ND & ND \\
\hline Number of genes & 12,409 & ND & ND & 11,873 & 11,865 \\
\hline GC content (\%) & 47.99 & 48.20 & 48.30 & 47.94 & 48.34 \\
\hline BUSCO completeness (\%) & 99.3 & ND & ND & 100 & 99.3 \\
\hline Origin & Rice, China, 2018 & Maize, Nepal, 1993 & Maize, Nepal, 1997 & Wheat, Brazil, 2012 & ND \\
\hline Reference & This study & Walkowiak et al. 2016 & Walkowiak et al. 2016 & Tralamazza et al. 2019 & ND \\
\hline
\end{tabular}

${ }^{\mathrm{a}} \mathrm{ND}=$ not described.

(15-ADON); and nivalenol (NIV) and its acetylated derivative, 4-acetyl nivalenol (4-ANIV) (Qiu et al. 2019). Among these fungi, F. meridionale is a serious yet comparatively understudied causal agent of FHB of wheat and rice; ear and stalk rot of maize; and pod discoloration, seed decay, and root rot of soybean (Chiotta et al. 2015; de Arruda et al. 2020; Kuhnem et al. 2016; Nerbass et al. 2015; Nicolli et al. 2015; Zhou et al. 2018). FHB of wheat caused by $F$. meridionale has been reported worldwide, including in Latin America (Aoki et al. 2012; de Arruda et al. 2020), Australia (Amarasinghe et al. 2019), China (Zhang et al. 2014), Nepal (Desjardins and Proctor 2011), and South Africa (Boutigny et al. 2011). In addition, $F$. meridionale has been found to be the predominant contributor and more competitive causal agent of ear rot of maize in Brazil (Machado et al. in press). Several studies showed that $F$. meridionale was frequently isolated from soybean in Argentina and Brazil (Barros et al. 2014; Chiotta et al. 2016). Recently, the endemic presence of $F$. meridionale causing head blight of rice has been reported in China (Dong et al. 2020) and in Brazil (Gomes et al. 2015). Based on results of the virulence assays, we found that $F$. meridionale strains could infect both wheat and rice, and were relatively more virulent in rice than in wheat compared with F. asiaticum and F. graminearum strains. (Dong et al. 2020) (F. Dong, X. Liu, Y. W. Lee, J. H. Xu, and J. R. Shi, unpublished data.). Moreover, a majority of $F$. meridionale isolates are NIV producers (Aoki et al. 2012; Scoz et al. 2009), with potentially higher levels of toxicity than other trichothecene FGSC producers (DON, 3-ADON, and 15-ADON) (de Arruda et al. 2020). Hence, obtaining high-quality genome sequences of $F$. meridionale would help expand current knowledge of the molecular mechanism underlying its pathogenesis as well as the genetic basis of its adaptation to different cereal hosts, which will be important for disease management and toxin contaminant control.

The first genome assemblies of $F$. meridionale were released for strains NRRL 28721 and NRRL 28723 isolated from maize in Nepal in 2016, strain Fmer152 isolated from wheat in Brazil in 2019, and strain CBS 110249 isolated from soil in South Africa. Based on Illumina sequencing, the genome assembly of maize $F$. meridionale strain NRRL 28271 has a total size of $36.5 \mathrm{Mb}$, with an $\mathrm{N}_{50}$ scaffold length of $0.11 \mathrm{Mb}$; that of NRRL 28723 is $36.4 \mathrm{Mb}$, with an $\mathrm{N}_{50}$ scaffold length of $0.41 \mathrm{Mb}$ (Walkowiak et al. 2016); that of wheat $F$. meridionale strain Fmer152 is $37.0 \mathrm{Mb}$, with an $\mathrm{N}_{50}$ scaffold length of $0.04 \mathrm{Mb}$ (Tralamazza et al. 2019); and that of soil F. meridionale strain CBS 110249 is $36.9 \mathrm{Mb}$, with an $\mathrm{N}_{50}$ scaffold length of 2.44 $\mathrm{Mb}$ (Table 1). However, these genomes are fragmented, because they were assembled using Illumina short reads, making it challenging to perform in-depth genomic analysis. To expand the genomic resources of $F$. meridionale from different hosts and improve the quality of $F$. meridionale genomic data, we used a combination of PacBio long-read sequencing and Illumina short-read sequencing technology to obtain the high-quality assembled genome of F. meridionale strain SR5, which was initially isolated from rice spikes in Sichuan Province of China in 2018 (Dong et al. 2020). Gene synteny was analyzed using TBtools and MCscanX (Chen et al. 2020; Wang et al. 2012). The results revealed an overall gene collinearity between $F$. meridionale SR5 and four other genome assemblies and a better completeness in SR5 assemblies with more continuous and detailed genomic sequences (Fig. 1).

Genomic DNA of $F$. meridionale SR5 extracted from mycelia harvested from a 3-dayold culture was used to construct a short-read library (an Illumina paired-end [PE] library) with a fragment size of approximately 350 bp and a PE 150-bp strategy using the Illumina TruSeq Nano DNA high-throughput library kit, and a long-read library (a PacBio 

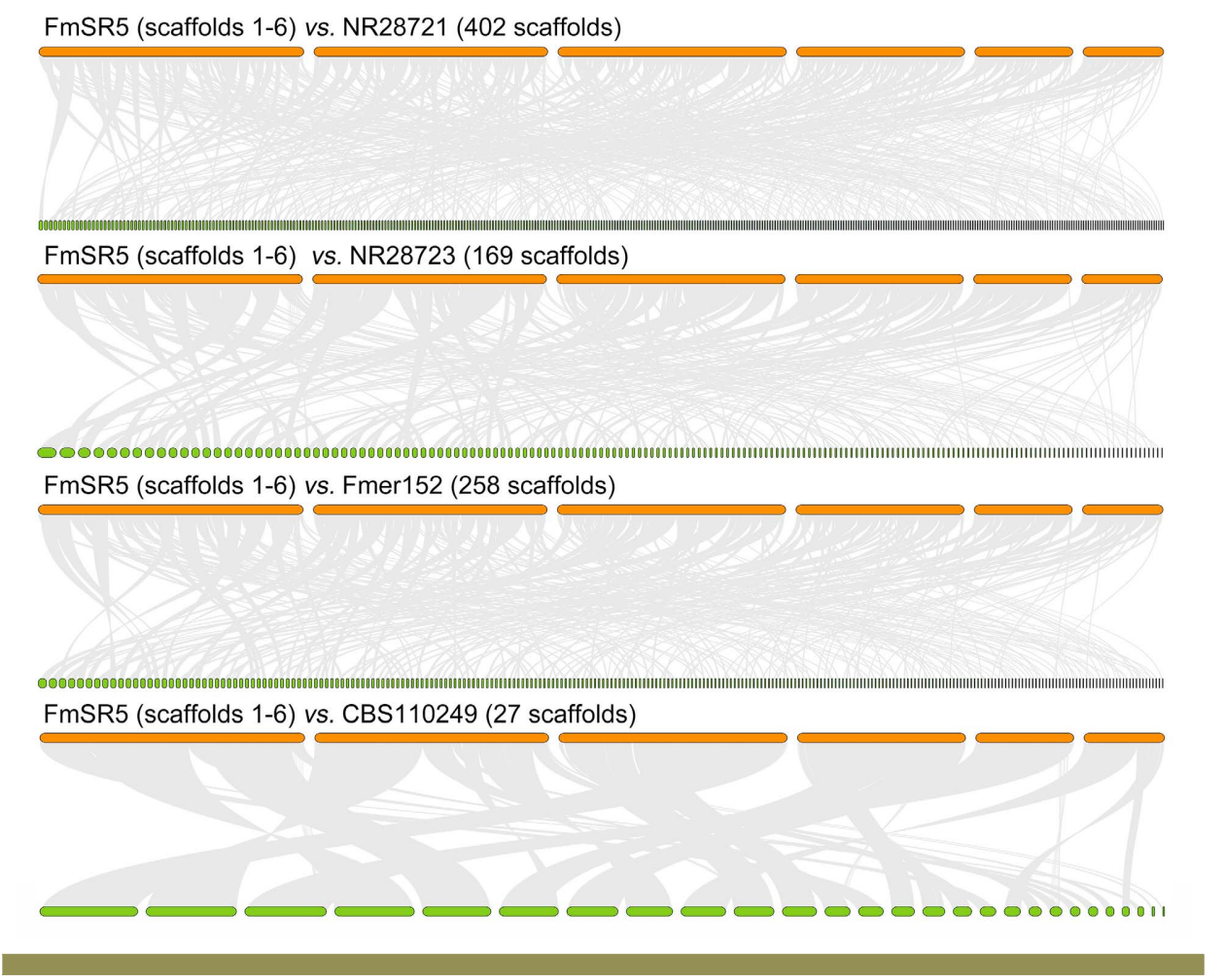

Fig. 1. Gene synteny between SR5 and the four other Fusarium meridionale assemblies.

Single-Molecule Real-Time [SMRT] library) with a fragment size of approximately $20 \mathrm{~kb}$ using the SMRT bell template prep kit 1.0 (PacBio). The Illumina PE library was sequenced using the Illumina HiSeq X Ten platform and resulted in $7.0 \mathrm{~Gb}$ of clean data (approximately 190x coverage), with 47.33 million clean reads generated. PacBio SMRT sequencing was performed using the PacBio Sequel System and resulted in a total of $14.29 \mathrm{~Gb}$ of clean data (approximately $388 \times$ coverage) and 1.12 million PacBio long reads, with a mean length of $12.7 \mathrm{~kb}$ and an $\mathrm{N}_{50}$ of $13.8 \mathrm{~kb}$.

De novo assembly was performed in Falcon (v0.3.0) using the clean PacBio long reads obtained from SMRT sequencing. Preassembled contigs were further corrected for random base error assessment by aligning the Illumina short reads using the Genome Analysis Toolkit (v1.6-13) (Broad Institute). Scaffolds were then constructed by SSPACE_Basic (v2.0) using the contigs, and gaps were filled by pbjelly2 (15.8.24). The final assembled genome of F. meridionale strain SR5 has a total size of $36.82 \mathrm{Mb}$, with nine scaffolds, a GC content of $47.99 \%$, and 12,409 predicted protein-encoding genes. The genome assembly has an $\mathrm{N}_{50}$ scaffold length of $7.81 \mathrm{Mb}$ (Table 1). The assembled genome of SR5 exhibits better continuity, as indicated by the increased scaffold $\mathrm{N}_{50}$ length (2.2- to 70-fold), and fewer scaffolds than the other three $F$. meridionale genomes (NR28721, NR28723, and Fmer152) reported to date (Table 1). The completeness of the SR5 genome assembly was analyzed using Benchmarking Universal Single-Copy Orthologs (BUSCOs, v4.14) (Simao et al. 2015). The analysis identified 288 (99.3\%) single-copy complete BUSCOs; only two in the 'fungi_odb9' data set were fragmented. According to a search for "TTAGGG", the repeat sequence widely found within the telomere structures, in the F. meridionale SR5 genome assembly, we found that all six of the largest scaffolds ( $36.77 \mathrm{Mb}, 99.9 \%$ of the full assembly) displayed telomere structures at one end and scaffold_3 at both ends.

AntiSMASH v4.1.0 identified 596 genes (4.8\%) in 43 predicted secondary metabolic gene clusters in the F. meridionale SR5 genome. The trichothecene biosynthetic gene cluster was predicted to have an intact TRI13 gene encoding the trichothecene C-4 hydroxylase required for NIV biosynthesis (Lee et al. 2002). Noncoding ribosomal RNA (rRNA) prediction was carried out by RNAmmer (v1.2) (Lagesen et al. 2007), transfer RNAs (tRNAs) were predicted with tRNAscan-SE (v1.3.1) (Lowe and Eddy 1997), and small RNAs (sRNAs) were identified using Infernal based on the Rfam database (v9.1) (Gardner et al. 2009). The F. meridionale SR5 genome is predicted to encode 68 rRNAs, 301 tRNAs, and 36 sRNAs. 
Repetitive elements (DNA transposons, tandem repeats, and interspersed elements) identified by RepeatMasker (v4-0-6) (Institute for Systems Biology) and Tandem Repeat Finder (v4.04) (Benson 1999) accounted for $2.26 \%$ of genomic sequences. In total, 12,409 genes were predicted, and functional annotation was performed using a combination of databases, including the NCBI Non-Redundant Protein database (12,292 genes, 99.05\%), Gene Ontology (6,875 genes, 55.4\%), Kyoto Encyclopedia of Genes and Genomes (4,594 genes, $37.02 \%)$, Swiss-Prot (3,460 genes, 27.88\%), Fungal Cytochrome P450 Database (2,159 genes, 17.39\%), and Carbohydrate-Active enZYmes Database (476 genes, 3.83\%).

The high-quality genome assembly of $F$. meridionale strain SR5 from rice provides valuable genomic resources for expanding our knowledge of Fusarium biology, pathogenesis, and pathogen-plant interaction mechanisms, thereby facilitating disease and toxin control.

\section{Data Availability}

The raw genome sequencing data, assembly, and annotated genes reported in this article have been deposited in the Genome Sequence Archive (accession ID CRA004534) and Genome Warehouse (accession ID GWHBDNV00000000) in the National Genomics Data Center of China and are publicly accessible online.

\section{Acknowledgments}

We thank the native English-speaking scientists of American Journal Experts (Durham, NC, U.S.A.) for editing our manuscript.

\section{Author-Recommended Internet Resources}

antiSMASH: https://antismash.secondarymetabolites.org

Carbohydrate-Active enZYmes Database: http://www.cazy.org

Falcon: https://github.com/PacificBiosciences/falcon

Kyoto Encyclopedia of Genes and Genomes: https://www.genome.jp/kegg

National Genomics Data Center of China: https://ngdc.cncb.ac.cn/gwh/

pbjelly2: https://sourceforge.net/projects/pb-jelly

SSPACE_Basic: https://www.baseclear.com/genomics/bioinformatics/basetools/SSPACE

\section{Literature Cited}

Amarasinghe, C., Sharanowski, B., and Fernando, W. G. D. 2019. Molecular phylogenetic relationships, trichothecene chemotype diversity and aggressiveness of strains in a global collection of Fusarium graminearum species. Toxins (Basel) 11:263.

Aoki, T., Ward, T. J., Kistler, H. C., and O'Donnell, K. 2012. Systematics, phylogeny and trichothecene mycotoxin potential of Fusarium head blight cereal pathogens. JSM Mycotoxins. 62:91-102.

Barros, G. G., Zanon, M. S. A., Chiotta, M. L., Reynoso, M. M., Scandiani, M. M., and Chulze, S. N. 2014. Pathogenicity of phylogenetic species in the Fusarium graminearum complex on soybean seedlings in Argentina. Eur. J. Plant Pathol. 138:215-222.

Benson, G. 1999. Tandem repeats finder: A program to analyze DNA sequences. Nucleic Acids Res. 27:573-580.

Boutigny, A. L., Ward, T. J., Van Coller, G. J., Flett, B., Lamprecht, S. C., O'Donnell, K., and Viljoen, A. 2011. Analysis of the Fusarium graminearum species complex from wheat, barley and maize in South Africa provides evidence of species-specific differences in host preference. Fungal Genet. Biol. 48:914-920.

Chen, C., Chen, H., Zhang, Y., Thomas, H. R., Frank, M. H., He, Y., and Xia, R. 2020. TBtools: An integrative toolkit developed for interactive analyses of big biological data. Mol. Plant 13:1194-1202.

Chiotta, M. L., Alaniz Zanon, M. S., Giaj-Merlera, G., Tessmann, D., Barros, G. G., and Chulze, S. 2015. Phylogenetic analyses of the Fusarium graminearum species complex isolated from soybean in Argentina and Brazil. Australas. Plant Dis. 10:32.

Chiotta, M. L., Alaniz Zanon, M. S., Palazzini, J. M., Scandiani, M. M., Formento, A. N., Barros, G. G., and Chulze, S. N. 2016. Pathogenicity of Fusarium graminearum and $F$. meridionale on soybean pod blight and trichothecene accumulation. Plant Pathol. 65:1492-1497.

de Arruda, M. H. M., Zchosnki, F. L., Silva, Y. K., de Lima, D. L., Tessmann, D. J., and Da-Silva, P. R. 2020. Genetic diversity of Fusarium meridionale, F. austroamericanum, and $F$. graminearum isolates associated with Fusarium head blight of wheat in Brazil. Trop. Plant Pathol. 46:98-108.

Desjardins, A. E., and Proctor, R. H. 2011. Genetic diversity and trichothecene chemotypes of the Fusarium graminearum clade isolated from maize in Nepal and identification of a putative new lineage. Fungal Biol. 115:38-48

Dong, F., Xu, J. H., Shi, J. R., Mokoena, M. P., Olaniran, A. O., Chen, X. Y., and Lee, Y.-W. 2020. First report of Fusarium head blight caused by Fusarium meridionale in rice in China. Plant Dis. 104:2726.

Gardner, P. P., Daub, J., Tate, J. G., Nawrocki, E. P., Kolbe, D. L., Lindgreen, S., Wilkinson, A. C., Finn, R. D., Griffiths-Jones, S., Eddy, S. R., and Bateman, A. 2009. Rfam: Updates to the RNA families database. Nucleic Acids Res. 37:D136-D140.

Gomes, L. B., Ward, T. J., Badiale-Furlong, E., and Del Ponte, E. M. 2015. Species composition, toxigenic potential and pathogenicity of Fusarium graminearum species complex isolates from southern Brazilian rice. Plant Pathol. 64:980-987.

Kuhnem, P. R., Ward, T. J., Silva, C. N., Spolti, P., Ciliato, M. L., Tessmann, D. J., and Del Ponte, E. M. 2016. Composition and toxigenic potential of the Fusarium graminearum species complex from maize ears, stalks and stubble in Brazil. Plant Pathol. 65:1185-1191.

Lagesen, K., Hallin, P., Rødland, E. A., Staerfeldt, H. H., Rognes, T., and Ussery, D. W. 2007. RNAmmer: Consistent and rapid annotation of ribosomal RNA genes. Nucleic Acids Res. 35:3100-3108.

Lee, T., Han, Y. K., Kim, K. H., Yun, S. H., and Lee, Y. W. 2002. Tri13 and Tri7 determine deoxynivalenol- and nivalenol-producing chemotypes of Gibberella zeae. Appl. Environ. Microbiol. 68:2148-2154.

Lowe, T. M., and Eddy, S. R. 1997. tRNAscan-SE: A program for improved detection of transfer RNA genes in genomic sequence. Nucleic Acids Res. 25:955-964.

Machado, F. J., Kuhnem, P., Casa, R. T., McMaster, N., Schmale, D., Vaillancourt, L. J., and Del Ponte, E. The dominance of Fusarium meridionale 
over F. graminearum causing Gibberella ear rot in Brazil may be due to increased aggressiveness and competitiveness. Phytopathology. In press.

Nerbass, F. R., Casa, R. T., Kuhnem, P. R., Vieira, J. A. L., and Valente, J. B. 2015. Field evaluation of maize for Gibberella ear rot resistance using silk channel and kernel inoculation with Fusarium meridionale. Trop. Plant Pathol. 40:388-393.

Nicolli, C. P., Spolti, P., Tibola, C. S., Fernandes, J. M. C., and Del Ponte, E. M. 2015. Fusarium head blight and trichothecene production in wheat by Fusarium graminearum and $F$. meridionale applied alone or in mixture at post-flowering. Trop. Plant Pathol. 40:134-140.

Qiu, J., Xu, J., and Shi, J. 2019. Fusarium toxins in Chinese wheat since the 1980s. Toxins (Basel) 11:248.

Scoz, L. B., Astolfi, P., Reartes, D. S., Schmale, D. G., Moraes, M. G., and Del Ponte, E. M. 2009. Trichothecene mycotoxin genotypes of Fusarium graminearum sensu stricto and Fusarium meridionale in wheat from southern Brazil. Plant Pathol. 58:344-351.

Simao, F. A., Waterhouse, R. M., loannidis, P., Kriventseva, E. V., and Zdobnov, E. M. 2015. BUSCO: Assessing genome assembly and annotation completeness with single-copy orthologs. Bioinformatics 31:3210-3212.
Tralamazza, S. M., Rocha, L. O., Oggenfuss, U., Corrêa, B., and Croll, D. 2019. Complex evolutionary origins of specialized metabolite gene cluster diversity among the plant pathogenic fungi of the Fusarium graminearum species complex. Genome Biol. Evol. 11:3106-3122.

Walkowiak, S., Rowland, O., Rodrigue, N., and Subramaniam, R. 2016. Whole genome sequencing and comparative genomics of closely related Fusarium Head Blight fungi: Fusarium graminearum, F. meridionale and F. asiaticum. BMC Genomics 17:1014.

Wang, Y., Tang, H., Debarry, J. D., Tan, X., Li, J., Wang, X., Lee, T. H., Jin, H., Marler, B., Guo, H., Kissinger, J. C., and Paterson, A. H. 2012. MCScanX: A toolkit for detection and evolutionary analysis of gene synteny and collinearity. Nucleic Acids Res. 40:e49.

Zhang, H., Luo, W., Pan, Y., Xu, J., Xu, J. S., Chen, W. Q., and Feng, J. 2014. First report of Fusarium maize ear rot caused by Fusarium meridionale in China. Plant Dis. 98:1156.

Zhou, D., Wang, X., Chen, G., Sun, S., Yang, Y., Zhu, Z., and Duan, C. 2018. The major Fusarium species causing maize ear and kernel rot and their toxigenicity in Chongqing, China. Toxins (Basel) 10:90. 